\title{
Community-level Educational Attainment and Dementia: A 6-year Longitudinal Multilevel Study in Japan
}

Tomo Takasugi ( $\nabla$ takasugi@hama-med.ac.jp )

Hamamatsu University School of Medicine

Taishi Tsuji

University of Tsukuba

Masamichi Hanazato

Chiba University

Yasuhiro Miyaguni

Nihon Fukushi University

Toshiyuki Ojima

Hamamatsu University School of Medicine

Katsunori Kondo

Chiba University

\section{Research Article}

Keywords: Socioeconomic status, education, cognitive decline, JAGES cohort, multilevel analysis

Posted Date: June 17th, 2021

DOl: https://doi.org/10.21203/rs.3.rs-608622/v1

License: (1) This work is licensed under a Creative Commons Attribution 4.0 International License.

Read Full License

Version of Record: A version of this preprint was published at BMC Geriatrics on November 23rd, 2021.

See the published version at https://doi.org/10.1186/s12877-021-02615-x. 


\section{Abstract}

\section{Background:}

Understanding of the association between community-level socioeconomic status and dementia is insufficient. We examined the contextual effect of community-level prevalence of lower educational attainment on the risk of dementia incidence. This work further explored the potential differences in associations for urban and non-urban areas.

\section{Methods:}

We analyzed a six-year prospective cohort data from the Japan Gerontological Evaluation Study, a nationwide survey between 2010 and 2012 of 49,888 (23,339 men and 26,549 women) physically and cognitively independent individuals aged $\geq 65$ years from 346 communities at 16 municipalities in seven prefectures. Dementia incidence was assessed through the public long-term care insurance system by the Ministry of Health, Labor, and Welfare in Japan. Educational years were dichotomized as $\leq 9$ years and $\geq 10$ years. We classified urban and non-urban areas using a Functional Urban Area defined by the European Union (EU) and Organisation for Economic Co-operation and Development (OECD). We performed a two-level (community- and individual-level) multilevel survival analysis to calculate hazard ratios (HRs) and 95\% confidence intervals (Cls).

\section{Results:}

The cumulative incidence of dementia during the follow-up period was $10.3 \%$. The mean proportion of educational attainment with $\leq 9$ years was $41.1 \%$ (range, $4.7 \%-88.4 \%$ ). Higher prevalence of communitylevel lower educational attainment had a significant association with a higher risk of dementia incidence (HR, 1.03; $95 \% \mathrm{Cl}, 1.00-1.06$, estimated by 10 percentage points of lower educational attainment) post adjusting for individual-level educational years, age, sex, income, residential years, disease, alcohol, smoking, social isolation, and population density. The association was significant in non-urban areas (HR, 1.05; 95\% Cl, 1.00-1.10).

\section{Conclusions:}

Older people living in communities with a higher prevalence of less educational attainment among their age demographic tended to develop dementia more often than those living in areas with a lower prevalence of less educational attainment after adjusting for individual-level educational attainment and covariates. In particular, the association was more pronounced in non-urban areas than in urban areas. Securing education for adolescence as a life course and population approach could thus be crucial to prevent dementia later in life among all older people living in non-urban areas.

\section{Background}


Dementia is a major global health issue due to rapid population aging worldwide, with over 50 million people being affected. The total number of people with dementia will reach 82 million in 2030 and 152 million in 2050 [1]. Mostly, half of the new 10 million dementia cases occur in Asia [2]. Dementia not only affects an individual's personal life, family, and career but also adds to high medical and social care costs [3].

A recent review reported that $40 \%$ of dementia cases are preventable, although there is currently no cure. The following 12 risk factors for dementia should be focused on: less education in early life, hearing loss, traumatic brain injury, hypertension, alcohol consumption, and obesity in midlife, smoking, depression, social isolation, physical inactivity, air pollution, and diabetes in late life. The second-highest percentage of these risk factors is less education [4]. A systematic review showed that lower education was associated with an increased risk for cognitive decline or dementia [5]. Recently, understanding the association between community-level, rather than individual-level, socioeconomic status (SES) and dementia and cognitive impairment is emphasized. For example, we can examine how community-level inequality of income affects individual-level dementia incidence after adjusting for individual-level SES. A systematic review found that lower community-level SES was associated with worse cognition [6]. Another review identified that living in urban areas was likely to reduce cognitive decline risk when compared to those in rural areas [7]. On the other hand, a recent US study showed that the prevalence of dementia declined more significantly among adults living in rural areas than those in urban areas over the past few decades as a result of improving educational attainment [8].

However, earlier studies might have four limitations relating to their research design. First, most were cross-sectional studies [6]. Second, the sample sizes in these studies were often limited to a few thousand participants [6]. Third, it was not common to adjust for confounding factors such as residential years $[6,9]$. Fourth, exploring the relationship between community-level educational attainment and dementia among urban and rural older adults was insufficient $[7,8]$. Overcoming the limitations of the aforementioned studies, this longitudinal study with large sample size data aimed to examine whether older populations living in communities with a higher proportion of lower educational attainment have the risk of dementia incidence when compared to those living in communities with a lower proportion of less education after controlling for individual-level educational years. We performed community- and individual-level multilevel analyses to investigate the contextual effect of community-level educational attainment on developing dementia among older individuals after adjusting for sufficient confounding factors. This work further explored the potential differences in associations for urban and non-urban areas.

\section{Methods}

\section{Participants}


This research was a six-year prospective longitudinal study with a large sample size data acquired from the Japan Gerontological Evaluation Study (JAGES). One objective of the JAGES is to identify social and behavioral factors associated with dementia onset among physically and cognitively independent individuals aged $\geq 65$ years $[10,11]$. A baseline mail self-reported questionnaire survey was administered from August 2010 to January 2012. In the study, 95,827 older people were chosen by random sampling from 16 municipalities in seven prefectures in Japan, including urban and non-urban areas. Among 62,426 respondents (response rate: $65.1 \%$ ), 56,687 participants had valid information on ID number, sex, and age (valid response rate: $59.2 \%$ ). To control for community-level educational attainment with a $\leq 9$ years variable, we excluded 2,666 respondents who lived in communities with less than 30 respondents and whose information regarding the extent of educational attainment and residence was unknown. Excluding these respondents resulted in 54,021 respondents living in 346 areas primarily defined by the school district. Of the 56,687 valid participants, 54,554 (96.2\%) respondents were successfully linked to the records of dementia incidence during a six-year follow-up term. A total of 49,888 participants $(23,339$ men and 26,549 women) were available for the present multilevel survival analyses (Figure 1). This sample size was determined after excluding survey respondents without information on the communitylevel variable (the extent of educational attainment) and those with limitations in basic activities of daily living, such as walking, bathing, and toileting, to ensure that the sample was both functionally and cognitively independent. Our research protocol and informed consent method were approved by the Human Subjects Committees of Nihon Fukushi University (no. 10-5) and the Chiba University Faculty of Medicine (no. 2493).

\section{Measures}

\section{Dependent Variable}

Our outcome was dementia incidence based on the public long-term care insurance records managed by the municipalities. The Ministry of Health, Labor, and Welfare (MHLW) in Japan established the Degree of Independency in Daily Lives of Demented Individuals [12]. In this system, a certification system in each local government sent personal investigators to applicants' homes to assess their nursing care eligibility (e.g., a home-helper). The investigators evaluated physical function, daily life ability, cognitive function, mental and behavioral disorders, adaptation to social life, and special medical treatment within 14 days $[13,14]$. Following the assessment, the investigators classified the applicants into eight ranks on a dementia scale according to their cognitive disability (Supplementary Table S1) [14, 15]. The dementia scale was highly correlated with the Mini Mental State Examination (Spearman rank correlation $\rho=$ -0.74) [16]. Moreover, another study showed that this dementia scale was a good indicator that reflects dementia diagnosis [17]. According to the MHLW, we defined rank II or above as dementia incidence showing some symptoms, behaviors, or communication problems during their daily lives [14, 18].

\section{Community- and Individual-level Independent Variables}

Participants were queried the following on educational attainment: "How many years of formal education have you had?" Answer choices included $<6,6-9,10-12$, and $\geq 13$ years. Educational attainment was 
dichotomized, separating those with $\leq 9$ years of education and those with $\geq 10$ years [19]. The cut-off point of educational attainment was set to 9 years because this has been the amount of compulsory education in Japan since 1947 [20]. We aggregated individual-level educational attainment by the community as the community-level independent variable.

\section{Covariates}

The following variables reported to be risk factors for dementia were evaluated as potential confounders in this study $[4,6]$. Data of age at baseline and sex were distributed based on the municipalities. Age was categorized into five groups: $65-69,70-74,75-79,80-84$, and $\geq 85$ years. As community-level covariates, the inhabitable area's population density in the participants' residential school districts was divided into quartiles $(\geq 10,100,7900-10,099,3280-7899$, or $<3280$ persons per square kilometer) [21]. We calculated an equivalized household income by dividing household income by the number of household members' square root. The income per year was categorized into three groups $(\leq 1.99,2-3.99$, or $\geq 4$ million yen) [18]. Years of residence were classified as seven groups $(<5,5-9,10-19,20-29,30-$ $39,40-49$, or $\geq 50$ years). Current medical treatment for those diseases known to raise the risk of dementia (stroke, hypertension, diabetes, and/or hearing loss) [4] was collected with yes/no answer choices. Health behaviors covered alcohol consumption (non, past, or current) and smoking status (non, past, or current). The definition of social isolation is social contact with other people less than once monthly [4]. We queried household members living with the participants and the frequency of seeing friends/acquaintances. In our study, socially isolated people were defined as those who live alone and meet with others less than monthly [21]. The individual-level covariates with missing data were assigned to a "missing" category. Therefore, participants with missing data on the covariates were included in the analysis.

\section{Classification of Urban and Non-Urban Areas}

We used the European Union (EU) and Organisation for Economic Co-operation and Development (OECD) definition of a Functional Urban Area (FUA). An FUA is a combination of multiple municipalities and consists of a city and its surroundings with less densely populated local units that are part of the commuting areas. FUAs are an excellent tool for comparing SES among cities [22]. FUAs are classified into the following four urban areas: 1) small urban areas with populations below 200,000;2) mediumsized urban areas with populations from $200,000-500,000 ; 3)$ metropolitan areas with populations from 500,000 to 1.5 million; and 4) large metropolitan areas with populations above 1.5 million [23]. In this study, the cut-off point of urban areas was set at a population with 500,000. Therefore, metropolitan areas and large metropolitan areas were categorized as "urban areas," and other areas were "non-urban areas" [24]. As a result, "urban areas" included five municipalities, and "non-urban areas" covered 11 municipalities.

\section{Statistical Analyses}


We performed a multilevel survival analysis incorporating both individual- and community-level factors. As recent research has reported that community-level factors had different impacts on urban and nonurban areas, we modeled urban and non-urban areas separately [8]. We calculated the hazard ratio (HR) and confidence interval $(\mathrm{Cl})$ for developing dementia during the follow-up term. The HR of communitylevel educational attainment was estimated as a 10 percentage point difference in aggregated educational attainment. Both community- and individual-level educational attainment and cross-level interaction terms were included in the crude model. In Model 1, the community-level covariate (population density) and individual-level covariates (age, sex, income, years of residence, stroke, hypertension, diabetes, hearing loss, drinking status, smoking status, and social isolation) were added. The significance level was set at less than $5 \%$ for all analyses. We used STATA/MP 16.1 (Stata Corp., College Station, TX) for all statistical analyses.

\section{Results}

The 49,888 participants in this study contributed to 263,419 person-years. In the study, 5,130 cases (10.3\%) acquired dementia during the follow-up period. The incidence per 1000 person-years was found to be 19.5 people. Table 1 describes all respondents' demographics and socioeconomic characteristics and those stratified by urban and non-urban areas. Overall, the majority of participants in non-urban areas had educational attainment of $\leq 9$ years; equivalized income of $\leq 1.99$ million yen; and were current drinkers. The proportion of educational attainment with $\leq 9$ years was calculated for each community. The mean (SD) and the ranges were $41.1 \%$ (17.5\%) and $4.7-88.4 \%$ (Table 2).

Table 3 shows the results of the multilevel survival analyses for incident dementia. According to the analysis for all participants, Model 1-including the community-level covariate and individual-level covariates and community-level higher prevalence of lower educational attainment-demonstrated a significant relationship with a higher risk of dementia ( $\mathrm{HR}, 1.03 ; 95 \% \mathrm{Cl}, 1.00-1.06)$, estimated by 10 percentage points of increment of a proportion of educational attainment in a community area. Individual-level educational attainment showed a significant association with a high likelihood of developing dementia (HR, 1.08; $95 \% \mathrm{Cl}, 1.01-1.16$ in Model 1). There were no significant cross-level interaction terms in any models.

In Model 1, for the non-urban area participants, community-level higher prevalence of lower educational attainment demonstrated a statistically significant relationship with a higher risk of dementia (HR, 1.05; $95 \% \mathrm{Cl}, 1.00-1.10)$. We found a statistically significant cross-level interaction term $(\mathrm{HR}, 0.94 ; 95 \% \mathrm{Cl}$, 0.88-0.999 in the crude model). This indicated that individuals with lower educational attainment declined $6 \%$ in dementia incidence, estimated by 10 percentage points of increment of a proportion of educational attainment in a community area. According to the analysis, for the participants in urban areas, the HRs of community-level higher prevalence of lower educational attainment were not significant in any of the models. In urban areas, individual-level educational attainment showed a significant relationship with a high likelihood of developing dementia (HR, 1.52; 95\% Cl, 1.40-1.65 in Crude Model). The significance disappeared in Model 1. 


\section{Discussion}

This is the first study to find the contextual effects of community-level prevalence of less educational attainment and developing dementia among older people using data based on a longitudinal and largescale sample. Older adults living in communities with a higher prevalence of less educational attainment among their age demographic tended to develop dementia more often than those living in areas with a lower prevalence of less educational attainment after adjusting for individual-level educational attainment and covariates. In particular, the association was more pronounced in non-urban areas than in urban areas.

Our results support the systematic review showing consistent evidence of an association between lower community-level SES, such as the proportion with no high school degree and worse cognition, after controlling for personal SES factors. A few longitudinal studies included in the review showed that community-level SES was significantly associated with cognitive decline. The proposed mediator between lower community-level SES and worse cognition may be social isolation [6]. In addition, previous studies have recommended that years of residence $[6,9]$ be included as potentially confounding factors. In our models, possible mediators and confounding factors, such as the abovementioned, were fully adjusted to overcome the existing studies' limitations. The current study was a longitudinal study with 50,000 participants, although the systematic review mainly included cross-sectional and small-sized studies of less than 10,000 [6]. Therefore, our study design was more robust than those of prior studies.

We found a contextual worse effect of community-level lower educational attainment on dementia incidence. People living in lower SES communities may heavily depend on community-level resources rather than individual-level ones due to the limitation of individual-level resources [25]. This means resources in the communities may affect the quality of life and health. Some disadvantages of lower SES communities (places with poor access, few bus lines, old-age income subsidies, and neighborhood organizations) were associated with lower cognitive decline [25, 26]. Moreover, previous studies have found that lower food store availability and lower sidewalk installation were associated with increased dementia incidence [14, 27]. Therefore, in the current study, lacking the physical and social resources in lower SES communities may increase dementia incidence when compared to higher SES communities.

Bridging social capital, which indicates a connection between different groups or SES levels, may be less developed in lower SES communities than in higher SES communities due to limited local resources. Therefore, the spreading of actions and information among people in lower SES communities may be inactive [28]. For example, people living in communities with stronger bridging of social capital may quickly acquire health or dementia prevention information through various local human networks. This idea is called "social contagion" [28, 29]. Moreover, the informal social control concept shows that people with higher social capital tend to work harder to maintain social order. These people ask people with unhealthy lifestyles, who include people with a lower SES, to change their health behaviors. In addition, people with unhealthy lifestyles can observe correct health behaviors and imitate their actions through community networks $[28,29]$. Therefore, improving bridging of social capital among older adults with a 
lower SES may result in a decline in the risk of dementia due to the effects of social contagion and informal social control.

On the other hand, there is strong bonding social capital in communities with a higher proportion of lower SES because people with a lower SES survive and help those with similar SES levels. Bonding social capital is associated with more psychological disorders [28]. This association may lead to raising the risk of dementia. In addition, because local resources are limited in lower SES communities when compared to higher SES communities, we may observe negative aspects of social capital, such as excessive demands on members, which leads to increased pain for a few limited, reliable members, and the exclusion of newcomers are residents with shorter years of residence [28]. The negative aspects of social capital may contribute to a higher risk of dementia. Development of human and economic resources, such as training community leaders and volunteer expenses, is simultaneously required in communities with more vital bonding social capital to reduce the negative impacts on social capital [28].

Our study is consistent with review articles that found that people living in urban centers tended to develop dementia less than those in rural areas [7,30]. Although there was an unequal distribution or a lack of resources related to better cognitive function (health clinics, bookstores, and libraries) due to the deficit of educated consumer demand or taxes for such resources in lower education communities [26], this may more easily happen in non-urban areas than in urban areas. Similarly, people living in lower education communities, especially non-urban areas, may be disproportionately exposed to chronic and stressful life conditions that generate hazards, such as decreased employment opportunities or income. This situation results in a lack of social resources (social clubs and neighborhood organizations) and safe physical resources, including gyms [26]. In the current study, few resources in non-urban areas may increase dementia incidence when compared to urban areas.

A systematic review with a meta-analysis concluded that early life rural living was strongly associated with a risk of Alzheimer's disease [30]. In addition, previous studies have mentioned that rural areas had educational inequality, such as more insufficient educational opportunities and less quality of education $[7,31]$. Although some children left rural areas and proceeded to higher grade schools in urban areas [31], many participants in the current study may live in the same rural areas where they received education for a long time [32]. A consequence of educational disadvantage in rural areas may cause an increase in the risk of dementia later in life [32], although we did not clarify the quality of education or the childhood residential areas in our study. On the other hand, a US study reported that cognitive decline was more significant among adults living in rural areas than those in urban areas during the past few decades. This resulted in a narrowing of the rural-urban disparities due to increasing education between 1910-1940 [8]. The same trend may occur in Japan in the future as compulsory education was extended from six to nine years in 1947 [20].

This study has some limitations. First, we did not identify transfers of residential areas, including urban to non-urban or non-urban to urban. In addition, we did not clarify the childhood residential areas among participants. However, we adjusted for years of residence to overcome the limitations of prior studies [6, 
9]. Second, we did not consider the quality of education. However, the majority of SES indicators in the existing studies were educational years [6]. Third, the data on some potentially community-level confounding factors, such as accessibility of local services, were lacking, although population density was taken into account.

\section{Conclusions}

Older people living in communities with a higher prevalence of less educational attainment among their age demographic tended to develop dementia more often than those living in areas with a lower prevalence of less educational attainment after adjusting for individual-level educational attainment and covariates. In particular, the association was more pronounced in non-urban areas than in urban areas. Securing education for adolescence as a life course and population approach could thus be crucial to prevent dementia later in life among all older people living in non-urban areas.

\section{List Of Abbreviations}

Cl: Confidence interval

EU: European Union

FUA: Functional Urban Area

HR: Hazard ratio

JAGES: Japan Gerontological Evaluation Study

MHLW: Ministry of Health, Labor, and Welfare

OECD: Organisation for Economic Co-operation and Development

SES: Socioeconomic status

\section{Declarations}

\section{Ethics approval and consent to participate}

The research protocol of JAGES and informed consent method were approved by the Human Subjects Committees of Nihon Fukushi University (no. 10-5) and the Chiba University Faculty of Medicine (no. 2493). We obtained written consent from the participants.

Consent for publication: Not applicable

Availability of data and materials 
Data are from the JAGES study. All enquiries are to be addressed at the data management committee via e-mail: dataadmin.ml@jages.net. All JAGES datasets have ethical or legal restrictions for public deposition due to inclusion of sensitive information from the human participants. Following the regulation of local governments that cooperated on our survey, the JAGES data management committee has imposed the restrictions upon the data.

\section{Competing interests}

The authors declare that they have no competing interests.

\section{Funding}

This study used data from JAGES (the Japan Gerontological Evaluation Study), which was supported by MEXT(Ministry of Education, Culture, Sports, Science and Technology-Japan)-Supported Program for the Strategic Research Foundation at Private Universities (2009-2013), JSPS(Japan Society for the Promotion of Science) KAKENHI Grant Numbers (JP18390200, JP22330172, JP22390400, JP23243070, JP23590786, JP23790710, JP24390469, JP24530698, JP24683018, JP25253052, JP25870573, JP25870881,JP26285138, JP26882010,JP15H01972 ), Health Labour Sciences Research Grants (H22Choju-Shitei-008, H24-Junkanki

[Seishu]- Ippan-007, H24-Chikyukibo-Ippan-009, H24-Choju-Wakate-009, H25-Kenki-Wakate-015, H25Choju-Ippan-003, H26-Irryo-Shitei-003 [Fukkou], H26-Choju-Ippan-006, H27-Ninchisyou-Ippan-001, H28choju-Ippan-002, H28- Ninchisyou-Ippan-002, H30-Kenki-Ippan-006, H30-Junkankitou-Ippan-004), Japan Agency for Medical Research and development (AMED) (JP17dk0110017, JP18dk0110027, JP18Is0110002, JP18le0110009), the Research Funding for Longevity Sciences from National Center for Geriatrics and Gerontology $(24-17,24-23,29-42,30-22)$. The views and opinions expressed in this article are those of the authors and do not necessarily reflect the official policy or position of the respective funding organizations.

\section{Authors' contributions}

TTa: conception, design, analysis and interpretation of the data, and writing the article; TTs: conception, design, interpretation of the data, and critical revision of the article; $\mathrm{MH}$ : conception, design, and critical revision of the article; YM: conception, design, and critical revision of the article; TO: critical revision of the article; and KK: conception, design, critical revision of the article, and principal investigator for the JAGES project. All authors read and approved the final draft submitted.

\section{Acknowledgements}

The authors would like to thank the study participants and the JAGES team for data collection. We would also like to thank members of the JAGES group for their advice about this study. 


\section{References}

1. Alzheimer's Disease International: World Alzheimer Report 2019.

2019. https://www.alz.co.uk/research/WorldAlzheimerReport2019.pdf. Accessed 2 March 2021.

2. Alzheimer's Disease International: World Alzheimer Report 2015.

2015. https://www.alz.co.uk/research/WorldAlzheimerReport2019.pdf. Accessed 2 March 2021.

3. World Health Organization: Demetia: Key facts. 2020. https://www.who.int/news-room/factsheets/detail/dementia. Accessed 2 March 2021.

4. Livingston G, Huntley J, Sommerlad A, Ames D, Ballard C, Banerjee S, Brayne C, Burns A, CohenMansfield J, Cooper C et al: Dementia prevention, intervention, and care: 2020 report of the Lancet Commission. The Lancet Commissions. 2020; 396(10248):413-446.

5. Harrison SL, Sajjad A, Bramer WM, Ikram MA, Tiemeier H, Stephan BCM: Exploring strategies to operationalize cognitive reserve: A systematic review of reviews. J Clin Exp Neuropsychol. 2015; 37(3):253-264.

6. Besser LM, McDonald NC, Song Y, Kukull WA, Rodriguez DA: Neighborhood Environment and Cognition in Older Adults: A Systematic Review. Am J Prev Med. 2017; 53(2):241-251.

7. Robbins RN, Scott T, Joska JA, Gouse H: Impact of urbanization on cognitive disorders. Current Opinion in Psychiatry. 2019; 32(3):210-217.

8. Weden MM, Shih RA, Kabeto MU, Langa KM: Secular Trends in Dementia and Cognitive Impairment of US Rural and Urban Older Adults. Am J Prev Med. 2018; 54(2):164-172.

9. Xiang $\mathrm{YX}$, Zare $\mathrm{H}$, Guan $\mathrm{CL}$, Gaskin $\mathrm{D}$ : The impact of rural-urban community settings on cognitive decline: results from a nationally-representative sample of seniors in China. BMC Geriatr. 2018; 18:323.

10. Kondo K, Rosenberg M, \& World Health Organization: Advancing universal health coverage through knowledge translation for healthy ageing: lessons learnt from the Japan gerontological evaluation study. 2018. https://apps.who.int/iris/handle/10665/279010. Accessed 2 March 2021.

11. Kondo K: Progress in Aging Epidemiology in Japan: The JAGES Project. Journal of Epidemiology. 2016; 26(7):331-336.

12. Tamiya N, Noguchi H, Nishi A, Reich MR, Ikegami N, Hashimoto H, Shibuya K, Kawachi I, Campbell JC: Population ageing and wellbeing: lessons from Japan's long-term care insurance policy. The Lancet. 2011; 378(9797):1183-1192.

13. Ministry of Health, Labour and Welfare: Textbook for Expert Investigators Concerning a Certification of Needed Long-term Care, Rvised Edition 2009. 2009. https://www.mhlw.go.jp/file/06- 
Seisakujouhou-12300000-Roukenkyoku/0000077237.pdf. Accessed 2 March 2021.

14. Tani Y, Suzuki N, Fujiwara T, Hanazato M, Kondo K: Neighborhood Food Environment and Dementia Incidence: the Japan Gerontological Evaluation Study Cohort Survey. Am J Prev Med. 2019; 56(3):383-392.

15. Hikichi H, Kondo K, Takeda T, Kawachi I: Social interaction and cognitive decline: Results of a 7year community intervention. Alzheimer's \& dementia. 2017; 3(1):23-32.

16. Hisano S: The relationship between Revised Hasegawa Dementia Scale (HDS-R), Mini-Mental State Examination (MMSE) and Bed-fast Scale, dementia scale. Jpn J Geriatr Psychiat. 2009; 20(8):883891.

17. Meguro K, Tanaka N, Kasai M, Nakamura K, Ishikawa H, Nakatsuka M, Satoh M, Ouchi Y:

Prevalence of dementia and dementing diseases in the old-old population in Japan: the Kurihara Project. Implications for Long-Term Care Insurance data. Psychogeriatrics. 2012; 12(4):226-234.

18. Takasugi T, Tsuji T, Nagamine Y, Miyaguni Y, Kondo K: Socioeconomic status and dementia onset among older Japanese: a six-year prospective cohort study from the Japan Gerontological Evaluation Study. Int J Geriatr Psychiatry. 2019; 34:1642-1650.

19. Basta NE, Matthews FE, Chatfield MD, Brayne C, Mrc C: Community-level socio-economic status and cognitive and functional impairment in the older population. Eur J Public Health. 2008; 18(1):48-54.

20. Ministry of Education, Culture, Sports, Science and Technology: Overview: Principles Guide Japan's Educational System. https://www.mext.go.jp/en/policy/education/overview/index.htm. Accessed 2 March 2021.

21. Tsuji T, Kanamori S, Miyaguni Y, Hanazato M, Kondo K: Community-Level Sports Group Participation and the Risk of Cognitive Impairment. Med Sci Sports Exerc. 2019; 51(11):2217-2223.

22. Dijkstra, L., Poelman, H., \& Veneri, P.: OECD Regional Development Working Papers 2019/11: The EU-OECD definition of a functional urban area. 2019. https://doi.org/10.1787/d58cb34d-en. Accessed 2 March 2021.

23. OECD: Redefining "Urban": A new way to measure metropolitan areas.

2012. https://doi.org/10.1787/9789264174108-en. Accessed 2 March 2021.

24. Ide K, Tsuji T, Kanamori S, Jeong S, Nagamine Y, Kondo K: Social Participation and Functional Decline: A Comparative Study of Rural and Urban Older People, Using Japan Gerontological Evaluation Study Longitudinal Data. Int J Environ Res Public Health. 2020; 17(2):617.

25. Luo Y, Zhang LL, Pan X: Neighborhood Environments and Cognitive Decline Among Middle-Aged and Older People in China. Journals of Gerontology Series B-Psychological Sciences and Social 
Sciences. 2019; 74(7):E60-E71.

26. Wight RG, Aneshensel CS, Miller-Martinez D, Botticello AL, Cummings JR, Karlamangla AS, Seeman TE: Urban neighborhood context, educational attainment, and cognitive function among older adults. Am J Epidemiol. 2006; 163(12):1071-1078.

27. Tani Y, Hanazato M, Fujiwara T, Suzuki N, Kondo K: Neighborhood sidewalk environment and incidence of dementia in older Japanese adults: the Japan Gerontological Evaluation Study cohort. Am J Epidemiol. 2021; kwab043.

28. Berkman LF, Kawachi I, Glymour MM: Social epidemiology (2nd ed.). New York: Oxford University Press; 2014.

29. Murayama H, Miyamae F, Ura C, Sakuma N, Sugiyama M, Inagaki H, Okamura T, Awata S: Does community social capital buffer the relationship between educational disadvantage and cognitive impairment? A multilevel analysis in Japan. BMC Public Health. 2019; 19:1442.

30. Russ TC, Batty GD, Hearnshaw GF, Fenton C, Starr JM: Geographical variation in dementia: systematic review with meta-analysis. Int J Epidemiol. 2012; 41(4):1012-1032.

31. Hendrie HC, Smith-Gamble V, Lane KA, Purnell C, Clark DO, Gao SJ: The Association of Early Life Factors and Declining Incidence Rates of Dementia in an Elderly Population of African Americans. Journals of Gerontology Series B-Psychological Sciences and Social Sciences. 2018; 73:S82-S89.

32. Saenz JL, Downer B, Garcia MA, Wong R: Cognition and Context: Rural-Urban Differences in Cognitive Aging Among Older Mexican Adults. J Aging Health. 2018; 30(6):965-986.

\section{Tables}

Table 1. Descriptive statistics of all individual-level variables 


\begin{tabular}{|c|c|c|c|c|c|c|}
\hline & \multicolumn{2}{|l|}{ Urban } & \multicolumn{2}{|c|}{ Non-Urban } & \multicolumn{2}{|l|}{ Total } \\
\hline Individual-level variables & $\mathbf{n}$ & $\%$ & $\mathbf{n}$ & $\%$ & $\mathbf{n}$ & $\%$ \\
\hline Total & 29,515 & $59.2 \%$ & 20,373 & $40.8 \%$ & 49,888 & $100.0 \%$ \\
\hline \multicolumn{7}{|l|}{ Dementia onset } \\
\hline No dementia & 26,685 & $90.4 \%$ & 18,073 & $88.7 \%$ & 44,758 & $89.7 \%$ \\
\hline Dementia & 2,830 & $9.6 \%$ & 2,300 & $11.3 \%$ & 5,130 & $10.3 \%$ \\
\hline \multicolumn{7}{|c|}{ Educational attainment (year) } \\
\hline$\leq 9$ & 12,554 & $42.5 \%$ & 12,113 & $59.5 \%$ & 24,667 & $49.4 \%$ \\
\hline$\geq 10$ & 16,961 & $57.5 \%$ & 8,260 & $40.5 \%$ & 25,221 & $50.6 \%$ \\
\hline \multicolumn{7}{|l|}{ Sex } \\
\hline Male & 14,051 & $47.6 \%$ & 9,288 & $45.6 \%$ & 23,339 & $46.8 \%$ \\
\hline Female & 15,464 & $52.4 \%$ & 11,085 & $54.4 \%$ & 26,549 & $53.2 \%$ \\
\hline \multicolumn{7}{|l|}{ Age } \\
\hline $65-69$ & 8,381 & $28.4 \%$ & 5,638 & $27.7 \%$ & 14,019 & $28.1 \%$ \\
\hline $70-74$ & 9,290 & $31.5 \%$ & 5,833 & $28.6 \%$ & 15,123 & $30.3 \%$ \\
\hline $75-79$ & 6,713 & $22.7 \%$ & 4,611 & $22.6 \%$ & 11,324 & $22.7 \%$ \\
\hline $80-84$ & 3,566 & $12.1 \%$ & 2,861 & $14.0 \%$ & 6,427 & $12.9 \%$ \\
\hline$\geqq 85$ & 1,565 & $5.3 \%$ & 1,430 & $7.0 \%$ & 2,995 & $6.0 \%$ \\
\hline
\end{tabular}

\section{Disease status in treatment}

\begin{tabular}{lllllll} 
Stroke: no & 28,647 & $97.1 \%$ & 19,682 & $96.6 \%$ & 48,329 & $96.9 \%$ \\
\hline Stroke: yes & 325 & $1.1 \%$ & 286 & $1.4 \%$ & 611 & $1.2 \%$ \\
\hline Hypertension: no & 17,393 & $58.9 \%$ & 11,728 & $57.6 \%$ & 29,121 & $58.4 \%$ \\
\hline Hypertension: yes & 11,579 & $39.2 \%$ & 8,240 & $40.4 \%$ & 19,819 & $39.7 \%$ \\
\hline Diabetes: no & 25,217 & $85.4 \%$ & 17,543 & $86.1 \%$ & 42,760 & $85.7 \%$ \\
\hline Diabetes: yes & 3,755 & $12.7 \%$ & 2,425 & $11.9 \%$ & 6,180 & $12.4 \%$ \\
\hline Hearing loss: no & 27,016 & $91.5 \%$ & 18,295 & $89.8 \%$ & 45,311 & $90.8 \%$ \\
\hline Hearing loss: yes & 1,956 & $6.6 \%$ & 1,673 & $8.2 \%$ & 3,629 & $7.3 \%$ \\
\hline Data Missing & 543 & $1.8 \%$ & 405 & $2.0 \%$ & 948 & $1.9 \%$
\end{tabular}


No

Yes

Data Missing

Drinking status

Non

Past

Current

Data Missing

Non

Past

Current

Data Missing

\section{Smoking status}

28,457

623

435
$96.4 \%$

$2.1 \%$

$1.5 \%$
19,753

298

322
$97.0 \%$

$1.5 \%$

$1.6 \%$
48,210

$96.6 \%$

$1.8 \%$

$1.5 \%$

\begin{tabular}{ll}
921 & $1.8 \%$ \\
\hline 757 & $1.5 \%$
\end{tabular}

$\begin{array}{llllll}10,092 & 34.2 \% & 6,000 & 29.5 \% & 16,092 & 32.3 \%\end{array}$

$\begin{array}{llllll}997 & 3.4 \% & 648 & 3.2 \% & 1,645 & 3.3 \%\end{array}$

$16,647 \quad 56.4 \% \quad 12,565 \quad 61.7 \% \quad 29,212 \quad 58.6 \%$

$\begin{array}{llllll}1,779 & 6.0 \% & 1,160 & 5.7 \% & 2,939 & 5.9 \%\end{array}$

$5.9 \%$

\begin{tabular}{lllllll} 
Non & 15,533 & $52.6 \%$ & 11,301 & $55.5 \%$ & 26,834 & $53.8 \%$ \\
\hline Past & 8,169 & $27.7 \%$ & 4,861 & $23.9 \%$ & 13,030 & $26.1 \%$ \\
\hline Current & 3,093 & $10.5 \%$ & 2,005 & $9.8 \%$ & 5,098 & $10.2 \%$ \\
Data Missing & 2,720 & $9.2 \%$ & 2,206 & $10.8 \%$ & 4,926 & $9.9 \%$
\end{tabular}

Equivalized income (million yen)

\begin{tabular}{lllllll}
$\leq 1.99$ & 11,008 & $37.3 \%$ & 9,179 & $45.1 \%$ & 20,187 & $40.5 \%$ \\
\hline $2-3.99$ & 10,418 & $35.3 \%$ & 5,663 & $27.8 \%$ & 16,081 & $32.2 \%$ \\
\hline 4 & 3,219 & $10.9 \%$ & 1,561 & $7.7 \%$ & 4,780 & $9.6 \%$ \\
\hline Data Missing & 4,870 & $16.5 \%$ & 3,970 & $19.5 \%$ & 8,840 & $17.7 \%$
\end{tabular}

Years of residence(year)

\begin{tabular}{lllllll}
$<5$ & 368 & $1.2 \%$ & 467 & $2.3 \%$ & 835 & $1.7 \%$ \\
\hline $5-9$ & 419 & $1.4 \%$ & 589 & $2.9 \%$ & 1,008 & $2.0 \%$ \\
\hline $10-19$ & 1,037 & $3.5 \%$ & 1,138 & $5.6 \%$ & 2,175 & $4.4 \%$ \\
\hline $20-29$ & 1,395 & $4.7 \%$ & 1,105 & $5.4 \%$ & 2,500 & $5.0 \%$ \\
\hline $30-39$ & 2,640 & $8.9 \%$ & 1,709 & $8.4 \%$ & 4,349 & $8.7 \%$ \\
\hline $40-49$ & 5,523 & $18.7 \%$ & 2,816 & $13.8 \%$ & 8,339 & $16.7 \%$ \\
\hline$\geq 50$ & 17,796 & $60.3 \%$ & 12,233 & $60.0 \%$ & 30,029 & $60.2 \%$ \\
\hline Data Missing & 337 & $1.1 \%$ & 316 & $1.6 \%$ & 653 & $1.3 \%$
\end{tabular}




\begin{tabular}{cllllll} 
Community-level Variables & $\mathbf{n}$ & $\%$ & $\mathbf{n}$ & $\%$ & $\mathbf{n}$ & $\%$ \\
\hline Total & 292 & $84.4 \%$ & 54 & $15.6 \%$ & 346 & $100.0 \%$
\end{tabular}

Proportion of Educational attainment

\begin{tabular}{|c|c|c|}
\hline Mean (SD) & $41.1 \%$ & $17.5 \%$ \\
\hline (Min-Max) & $(4.7 \%$ & $8.4 \%)$ \\
\hline
\end{tabular}

Population density (persons per square $\mathrm{km}$ of inhabitable area)

Highest quartile $(\geq 10,100) \quad 86$

Second quartile $(7900-10,099) \quad 87$

Third quartile (3280-7899) $\quad 86$

Lowest quartile $(<3280) \quad 87$

Table 3 Results of multilevel survival analysis for developing dementia (participants nested in 346 community areas) 
Crude

$\mathrm{HR} \quad 95 \% \mathrm{Cl}$
Model 1

$\mathrm{HR} \quad 95 \% \mathrm{Cl}$

\section{All participants $(N=49,888)$}

\begin{tabular}{|c|c|c|c|c|c|c|}
\hline Community-level educational attainment ${ }^{a}$ & 1.04 & $(1.01-1.06)$ & * & 1.03 & $(1.00-1.06)$ & * \\
\hline Individual-level educational attainment ${ }^{b}$ & 1.55 & $(1.44-1.66)$ & * & 1.08 & $(1.01-1.16)$ & * \\
\hline Cross-level interaction & 0.97 & $(0.93-1.01)$ & & 0.99 & $(0.95-1.02)$ & \\
\hline \multicolumn{7}{|l|}{ Non-Urban $(n=20,373)$} \\
\hline Community-level educational attainment ${ }^{\mathrm{a}}$ & 1.07 & $(1.02-1.12)$ & * & 1.05 & $(1.00-1.10)$ & * \\
\hline Individual-level educational attainment ${ }^{\mathrm{b}}$ & 1.67 & $(1.44-1.93)$ & * & 1.07 & $(0.93-1.24)$ & \\
\hline Cross-level interaction & 0.94 & $(0.88-0.999)$ & * & 0.99 & $(0.93-1.05)$ & \\
\hline \multicolumn{7}{|l|}{ Urban $(n=29,515 \square$} \\
\hline Community-level educational attainment ${ }^{a}$ & 1.00 & $(0.97-1.04)$ & & 1.02 & $(0.98-1.05)$ & \\
\hline Individual-level educational attainment ${ }^{\mathrm{b}}$ & 1.52 & $(1.40-1.65)$ & * & 1.08 & $(0.998-1.18)$ & \\
\hline Cross-level interaction & 0.97 & $(0.91-1.03)$ & & 0.99 & $(0.93-1.04)$ & \\
\hline
\end{tabular}

Notes: HR: hazard ratio; $\mathrm{Cl}$; confidence interval

${ }^{a} H R$ for 10 percentage point increment of proportion of educational attainment in a community area.

${ }^{\mathrm{b}} \mathrm{HR}$ for educational attainment less than $9 \mathrm{yr}$.

Model 1: crude model + age + sex + community-level covariate (population density) + individual-level covariates (income, years of residence, stroke, hypertension, diabetes, hearing loss, drinking status, smoking status, and social isolation).

$*: p<0.05$

\section{Figures}




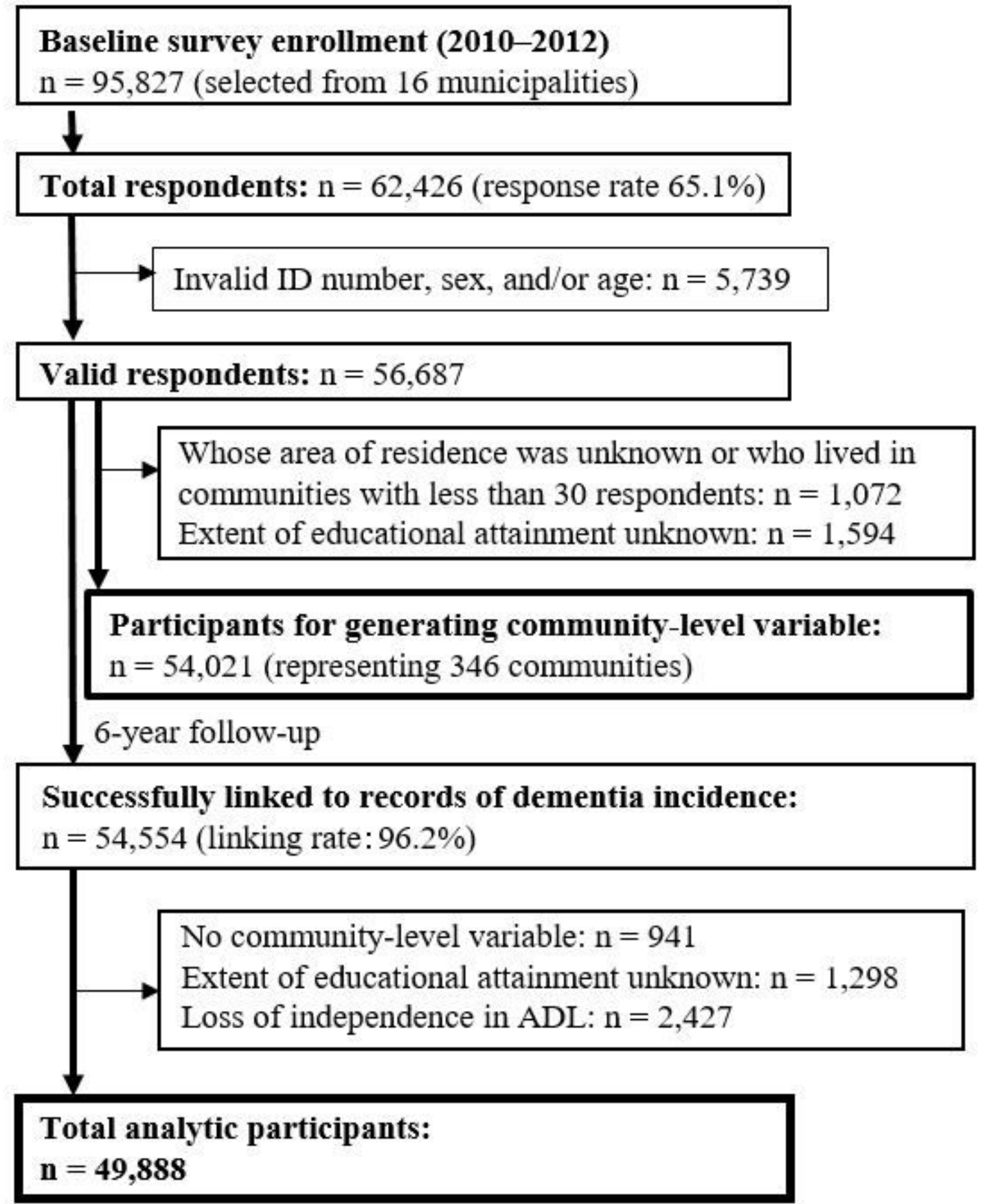

Figure 1

Flow of participants in the cohort study.

\section{Supplementary Files}

This is a list of supplementary files associated with this preprint. Click to download.

- SupplementaryTableS1.docx 